Ocula ${ }^{15}$

Commemorating Charles S. Peirce (1839-1914):

interpretive semiotics and mass media

A cura di Giampaolo Proni e Salvatore Zingale

Novembre 2014 I www.ocula.it - DOI: 10.12977/ocula31

\title{
Note per una tipologia dicisegnica
}

\author{
Valerio De Michele \\ Università di Bologna \\ valeriodemichele@libero.it
}

\begin{abstract}
Nella Speculative Grammar, Peirce propone due tipologie dicisegniche: dicisegni indicali e dicisegni Simbolici.

La loro architettura sembra ricalcare quella dei relativi completi presentati nella Logica del 1897. In entrambi i casi abbiamo, infatti, un'icona che presenta dei punti vuoti. Un'icona che scava nella pienezza del proprio assetto il posto di una mancanza, la necessità di inerire a un "altro". I Dicisegni presentano quindi una PARTE ICONICA (che si offre come strutturalmente autonoma, logicamente completa) sulla quale si innesta Una PARTE INDICALE (che esibisce la propria strutturale incompletezza, la necessità di inerire a un'entità altra).

Secondo la mia lettura, le due tipologie dicisegniche individuano due differenti modi di saturare la PARTE INDICALE di questa struttura profonda.

Nei Dicisegni Indicali, la parte indicale viene saturata attraverso il ricorso a un'unità semiosica di natura ancora indicale. Il dicisegno mette in scena una predicazione che si applica a un'entità, la quale trova espressione, all'interno del segno, in modo indicale. Nei Dicisegni Simbolici, la parte indicale viene saturata attraverso il ricorso a un'unità semiosica di natura simbolica. Il dicisegno mette in scena una predicazione che si applica a un'entità, la quale trova espressione, all'interno del segno, in modo simbolico. Il modello peirciano appare incompleto per due ragioni: i) si concentra sulla necessità di saturare solo la PARTE INDICALE del dicisegno, tralasciando del tutto la PARTE ICONICA; ii) si concentra solo su una saturazione indicale e simbolica della PARTE INDICALE, tralasciando una possibile saturazione iconica.

Tento quindi di ricondurre le due tipologie proposte da Peirce a un modello più generale, nel quale: i) si prende in considerazione tanto la parte indicale che iconica del dicisegno e, di seguito, ii) si cerca di dare fondo a tutte le loro possibili saturazioni: iconiche, indicali, simboliche. Ne nasce una combinatoria che permette di individuare nove differenti tipologie dicisegniche.
\end{abstract}

\section{Parole chiave}

Dicisegno, Icona, Indice, Simbolo, Speculative Grammar

\section{Sommario}

1. Introduzione

2. Architettura generale dei dicisegni

3. Le tipologie dicisegniche della Speculative Grammar

4. Le tipologie dicisegniche. Dicisegni Indicali vs Dicisegni Simbolici

5. Rilancio del modello peirciano

6. Conclusione

Bibliografia 


\section{Ocula $a^{15}$}

Commemorating Charles S. Peirce (1839-1914): interpretive semiotics and mass media

Valerio De Michele, Note per una tipologia dicisegnica

\section{Introduzione}

La sezione dei Collected Papers intitolata Speculative Grammar riunisce otto scritti peirciani risalenti a epoche molto diverse. On reasoning in gener$a l$, il più vecchio, è del 1893; Meaning, il più recente, risale al 1910.

La parte più consistente, circa 27 paragrafi sui 76 complessivi, è occupata da un manoscritto del 1903, intitolato Nomenclature and Divisions of triadic relations, as far as they are determined. Qui Peirce tenta di indagare il meccanismo triadico del segno osservandolo da tre diverse prospettive. Taglia la presupposizione reciproca tra Representamen-Oggetto-Interpretante, la necessaria co-implicazione logica delle polarità semiotiche, secondo tre diversi piani: i) secondo il possibile modo d'essere del segno in sé; ii) secondo il possibile modo in cui il Representamen può rapportarsi all'Oggetto; iii) secondo il possibile modo in cui l'Interpretante può raffigurare il Representamen.

Ogni livello individua tre possibilità. L'analisi produce, quindi, tre diverse tricotomie: i) Qualisegno-Sinsegno-Legisegno; ii) Icona-Indice-Simbolo; iii) Rema-Dicisegno-Argomento.

Dalla combinazione delle tre tricotomie nascono dieci possibili tipi di segno; dieci possibili modalità della semiosi.

L'obiettivo è quello di gettare le basi per una scienza generale della significazione; fornire strumenti per indagare le diverse modalità con cui un segno può rinviare a qualsiasi tipo di significato. Rifacendosi alla Grammatica Speculativa di Tommaso di Erfurt (testo all'epoca attribuito a Duns Scoto), Peirce chiama questa branca della semiotica Grammatica Pura:

La scienza della semiotica ha tre branche. La prima è chiamata da Duns Scoto grammatica speculativa; possiamo chiamarla grammatica pura: il suo compito è di accertare che cosa deve essere vero dei representamen usati da ogni intelletto scientifico affinché essi siano atti a veicolare qualsiasi significato. (CP 2.229)

In questo saggio mi concentrerò sulla terza tricotomia. In particolare, proverò a indagare i dicisegni. Cercherò di individuare nuove tipologie dicisegniche che possano affiancare quelle indicate da Peirce. Si tratta, quindi, di una ideale integrazione del testo peirciano; del tentativo di spostare di qualche passo l'orizzonte della Grammatica Speculativa.

\section{Architettura generale dei dicisegni}

I dicisegni sono segni informativi: dicono qualcosa di qualcosa. Attribuiscono qualcosa (il predicato) a qualcosa (il soggetto). In sintesi, mettono in scena una predicazione, nella quale la copula salda allo stesso tempo la differenza e la coesione tra ciò che si dice e ciò di cui si dice.

L'architettura dei dicisegni ricalca quella dei Relativi Completi descritti da Peirce nella logica dei relativi del 1897. Abbiamo un'icona che presenta dei punti vuoti. Un'icona che scava nella pienezza del proprio assetto il posto di una mancanza, la necessità di inerire a un "altro". Abbiamo, insomma, uno spazio iconico, uno spazio che si offre come strutturalmente autonomo, com- 


\section{Ocula $a^{15}$}

Commemorating Charles S. Peirce (1839-1914): interpretive semiotics and mass media

Valerio De Michele, Note per una tipologia dicisegnica

pleto nella propria architettura logica, sul quale si innesta uno spazio indicale, uno spazio che esibisce la propria costitutiva incompletezza, la necessità di integrare un'entità altra.

L'architettura profonda del dicisegno presenta così una PARTE ICONICA, intesa come logicamente completa, alla quale si applica una PARTE INDICALE, intesa come logicamente incompleta (fig.1).

LIVELLO SUPERFICIALE

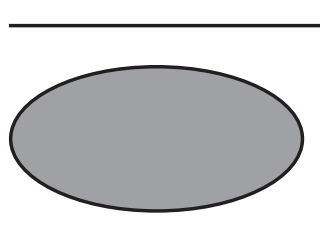

Icona

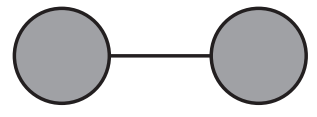

Indice

Figura 1. Struttura profonda del Dicisegno.

\section{Le Tipologie dicisegniche della Speculative Grammar}

Nella Speculative Grammar, Peirce individua due tipologie dicisegniche: i) Dicisegni indicali (di tipo sinsegnico o legisegnico); ii) Dicisegni simbolici (di tipo necessariamente legisegnico). Secondo la mia lettura, queste due tipologie individuano due differenti modi di esprimere l'architettura profonda del dicisegno.

Nei Dicisegni indicali, la parte indicale e la parte iconica, che costituiscono l'architettura profonda, vengono entrambe espresse attraverso entità semiosiche di natura indicale.

Nei Dicisegni simbolici, la parte indicale e quella iconica di base trovano espressione, invece, attraverso il ricorso a unità di natura simbolica.

Prima di passare in rassegna, concretamente, queste due tipologie segniche, credo sia necessario un rapido excursus sulla nozione generale di indice. Come vedremo, infatti, la distinzione tra indici genuini e indici degenerati si rivelerà essenziale per comprendere la differenza tra dicisegni indicali di tipo sinsegnico e dicisegni indicali di tipo legisegnico.

\subsection{Note sul rinvio indicale: indici genuini vs indici degenerati}

La relazione indicale salda la coesione tra due differenti porzioni dell'esperienza. Fa in modo che l'una sia ciò che l'altra la fa essere. Che l'una non possa, quindi, essere concepita senza l'altra. Che possa, di seguito, rinviare all'altra in quanto ne risulta interamente determinata.

Per spiegare questa relazione, Peirce ricorre, nella lettera a Calderoni sul pragmaticismo, a un esempio caro alla filosofia Scolastica, quello del rapporto padre/figlio: "Un padre non è un padre se suo figlio muore, e non ha quel modo d'essere che aveva quando il figlio era vivo" (CP 8.213). 


\section{Ocula $a^{15}$}

Commemorating Charles S. Peirce (1839-1914): interpretive semiotics and mass media

Valerio De Michele, Note per una tipologia dicisegnica

Nel caso degli indici genuini, questo rapporto di co-implicazione logica si stabilisce di fatto e interessa entità individuali. L'esempio tipico è quello della causalità fisica che unisce l'impronta all'impressore.

Nel caso degli indici degenerati (ipoindici), la co-implicazione logica ha carattere convenzionale e chiama in causa unità replicabili. L'esempio tipico è costituito dai dimostrativi, dai deittici e dai nomi propri:

I Subindici o iposemi sono segni che sono resi tali principalmente in virtù di una connessione attuale con i loro oggetti. Così, un nome proprio, o un dimostrativo personale, o un pronome relativo, o la lettera che contrassegna un diagramma denota ciò che denota grazie a una connessione attuale col suo oggetto, ma nessuno di essi è un indice vero e proprio dal momento che non è un individuo. (CP 2.284)

Si tratta di unità linguistiche che non sono legate a significati generici, ma puntano a unità semiosiche individuali (una situazione, una persona) e si offrono come se ne fossero direttamente causate.

Cerchiamo di comprendere meglio in che cosa la connessione attuale, tipica dei subindici, differisce dalla connessione reale, tipica degli indici genuini.

Partiamo dalle somiglianze. Tanto gli indici genuini che degenerati individuano una relazione semiosica. Mettono in scena, quindi, un meccanismo necessariamente triadico. In entrambi i casi, il segno, per poter rinviare al proprio oggetto, dovrà fare riferimento a un terzo attante: un interpretante che ha la funzione di individuare il carattere rappresentativo alla base del rinvio; di delineare la ratio che, segnando la reciproca commensurabilità dei relati, spiega il possibile passaggio dall'uno all'altro.

Detto questo, la differenza tra indici genuini e degenerati sembra risiedere nel modo in cui tale meccanismo si autorappresenta. In particolare, la differenza sta nel modo in cui l'interpretante descrive il proprio ingaggio e il proprio rapporto con le altre due polarità attanziali.

Negli indici genuini, la relazione indicale, la co-implicazione logica tra representamen e oggetto, viene intesa come precedente e autonoma rispetto al momento interpretativo che la rileva. L'interpretante si offre, quindi, come la scoperta di un meccanismo già esistente.

Nei subindici, la relazione tra i funtivi viene intesa, invece, come successiva e dipendente rispetto al momento interpretativo che la rileva. L'interpretante si offre, quindi, come l'invenzione di un meccanismo prima non esistente.

Riassumendo, la distinzione tra indici genuini e degenerati sembra afferire a due differenti modi in cui l'interpretante indicale può rendere conto del proprio funzionamento, esibendo la propria priorità o posteriorità logica rispetto all'asse Representamen-Oggetto. La distinzione non riguarda il funzionamento logico della semiosi, ma le modalità stilistiche che esso può adottare per autorappresentarsi.

\subsubsection{Termometri}

Prendiamo il caso di un termometro. La relazione indicale tra il livello del mercurio e il livello della temperatura è genuina. Affinché tale rapporto si renda intelligibile, affinché emerga nel proprio funzionamento semiosico, è necessario 


\section{Ocula $a^{15}$}

Commemorating Charles S. Peirce (1839-1914): interpretive semiotics and mass media

Valerio De Michele, Note per una tipologia dicisegnica

l'intervento di una mente interpretante. Il meccanismo di causazione può darsi come tale solo per qualcuno. È solo in relazione a un'istanza interpretativa (interpretante-terzo) che il mercurio può assumere la sua funzione di attante-causato (representamen-primo) indicalmente indirizzato verso un attante-causante (oggetto-secondo), costituito, in questo caso, dal livello di temperatura.

Allo stesso tempo, la relazione indicale si offre all'interprete come perfettamente efficiente e conclusa prima e oltre il suo intervento. In altri termini, l'attivazione dell'istanza interpretativa, per quanto, come si è visto, essenziale alla costituzione di qualsiasi nesso indicale, si sviluppa in questi casi secondo una particolare modalità stilistica che ne occulta il carattere fondante e la restituisce come un momento teorico successivo e non necessario. L'interpretante del rapporto causale si offre quindi come una istanza di riscrittura, come la sistemazione successiva di una dinamica già perfettamente autonoma. Insomma, per quanto sia necessario un interpretante affinché il rapporto di causazione tra mercurio e temperatura possa emergere, l'interprete del termometro sa che tale rapporto non attende il suo sguardo per seguire il proprio corso.

Riassumendo, in questi casi l'azione dell'interpretante si offre come un momento accessorio, non fondante rispetto al rapporto che individua. Come un momento di scoperta, non di invenzione.

L'istanza interpretante di un indice genuino sembra prevedere, come componente integrante del proprio ingaggio, la negazione della propria pertinenza. In questo senso la co-determinazione logica tra representamen e oggetto viene intesa come precedente e autonoma rispetto al momento interpretativo che la rileva.

\subsubsection{Cestini}

Prendiamo il caso dell'avviso "gettare qui la carta", affisso su un cestino per la raccolta. La relazione indicale tra "qui" (unità semiosica legisegnica) e il cestino (unità semiosica percettiva) è degenerata. Ancora una volta, il rapporto indicale tra le due unità può strutturarsi solo grazie all'intervento di una mente interpretante. È solo grazie all'attivazione di una specifica istanza interpretativa che il "qui" può assumere la funzione di un indice-rinviante puntato verso un indice-rinviato, nel nostro caso costituito dal cestino.

Diversamente dal caso del termometro, la relazione indicale si offre ora all'interprete come direttamente istituita dal suo intervento. L'attivazione dell'istanza interpretativa si sviluppa secondo una particolare modalità stilistica che ne evidenza il carattere fondante e la restituisce come un momento teorico necessario e precedente al funzionamento del nesso indicale. L'interpretante dell'indice si offre come un'istanza creatrice che provvede a strutturare una relazione prima inesistente. Insomma, l'interprete del cartello sa che il rapporto tra la parola e il cestino esiste solo grazie al suo sguardo.

Riassumendo, l'azione dell'interpretante si offre ora come un momento che, oltre a rilevare la relazione semiosica, provvede anche a generarla. Un momento di invenzione, quindi, non di scoperta.

Per questa ragione la co-determinazione logica tra representamen e oggetto viene intesa come successiva e direttamente dipendente rispetto al momento interpretativo che la individua. 


\section{Ocula $a^{15}$}

Commemorating Charles S. Peirce (1839-1914): interpretive semiotics and mass media

Valerio De Michele, Note per una tipologia dicisegnica

In conclusione, secondo il sistema peirciano, tutto ciò che risulta concepibile lo è in forma semiosica; il pensiero, quindi, lavora necessariamente con segni:

Noi pensiamo soltanto per segni. Questi segni mentali sono di natura mista; le parti-simbolo di essi sono chiamate concetti. Se un uomo crea un nuovo simbolo, ciò accade attraverso pensieri che implicano concetti. Così un nuovo simbolo può germinare solo da simboli. Omne symbolum de symbolo. [...] il simbolo può, con la sfinge di Emerson, dire all'uomo: dell'occhio tuo io sono il raggio. (CP 2.302)

Le relazioni indicali genuine individuano il luogo teorico nel quale, a partire dalla terzità (da cui non è mai concesso uscire), diviene possibile intravedere la secondità; il regno delle relazioni di forza bruta, dei rapporti di azione/ reazione che ci appaiono inevitabilmente ciechi, privi di ogni relazione a un terzo (inteso come finalità o significatività). La Seconditas deve essere intesa, quindi, come un effetto di senso del triadismo semiosico. È la conseguenza del modo in cui l'interpretante rappresenta il proprio ingaggio; del suo porsi come logicamente successivo rispetto al rapporto representamen-oggetto.

Le relazioni degenerate, invece, individuano il luogo teorico nel quale il perfetto equilibrio tra le istanze semiosiche cede il passo, stilisticamente, alla priorità dell’interpretante rispetto alla relazione representamen-oggetto.

\section{Le tipologie dicisegniche. Dicisegni Indicali vs Dicisegni Simbolici}

Chiarito il generale funzionamento degli indici e le ragioni della differenza tra quelli genuini e degenerati, passiamo finalmente allo studio delle due tipologie dicisegniche presentate nella Speculative Grammar.

\subsection{I dicisegni indicali}

I dicisegni indicali sono segni che forniscono informazioni riguardo un oggetto al quale risultano indicalmente connessi. Nei dicisegni indicali sinsegnici, la connessione indicale tra segno e oggetto è reale, genuina; in quelli legisegnici, invece, è attuale, degenerata.

\subsubsection{Dicisegni indicali sinsegnici}

Due tipici esempi di dicisegni indicali sinsegnici sono rappresentati dalle fotografie istantanee e dalle bandierine utilizzate per segnalare la direzione del vento.

La fotografia è il residuo di un processo foto-chimico, la traccia di una frizione interna alla sfera del visibile. In quanto direttamente plasmata dal proprio oggetto, secondo individuale di un rapporto di causazione, la fotografia vale come indice genuino:

La fotografia, specialmente le istantanee, sono molto istruttive, perché sappiamo che per certi aspetti esse sono esattamente uguali agli oggetti che esse rappresentano. Ma questa rassomiglianza è dovuta al fatto che le fotografie sono state prodotte in condizioni tali che esse erano fisicamente costrette a corrispondere punto per punto all'oggetto in natura. (CP 2.281) 


\section{Ocula $a^{15}$}

Commemorating Charles S. Peirce (1839-1914): interpretive semiotics and mass media

Valerio De Michele, Note per una tipologia dicisegnica

Si tratta di un indice altamente istruttivo, informativo:

Il fatto che la seconda [la fotografia] sia considerata l'effetto delle radiazioni di luce provenienti dall'oggetto la rende un indice, e un indice altamente informativo. (CP 2.265)

L'oggetto imprime, infatti, nella pasta fotosensibile il calco del proprio sembiante visivo, la traccia di molti suoi caratteri percettivi. Partendo da queste marche indicalmente impresse, la fotografia consente di recuperare un frammento della vita percettiva dell'oggetto, di ricostruire alcuni dei suoi caratteri visivi.

Per questa ragione la fotografia può funzionare come un segno informativo, come un dicisegno. Permette di predicare diverse qualità del proprio oggetto. Consente di dire: "Quello (soggetto) era così e così (predicato)".

La particolare modalità di produzione del segno fotografico fa in modo che tanto la parte indicale, quanto la parte iconica della struttura profonda del dicisegno; tanto ciò che si dice (il predicato), quanto ciò di cui si dice (il soggetto), siano presenti, nel segno, come fasci di indici. In altri termini, sia le caratteristiche che verranno attribuite, sia il soggetto al quale esse verranno attribuite, sono presenti nel segno sotto forma di tratti indicali: come tracce di una catena di costrizioni fisiche.

Andrebbe precisato che, nel particolarissimo caso delle fotografie, l'oggetto trova posto nel segno solo attraverso i caratteri che riesce indicalmente a imprimere. Di conseguenza, l'indice, che nel segno ha la funzione di individuare il soggetto della predicazione, non può essere distinto dall'insieme degli indici che individuano le caratteristiche che potranno essergli attribuite. Il soggetto è dato dalla somma degli attributi che riesce a imprimere e che potranno, di seguito, essergli predicati.

Risulta così impossibile distinguere di fatto, nella fotografia, il posto del soggetto e quello del predicato. Il primo si delinea, in filigrana, attraverso il secondo.

Tuttavia, come cercherò di fare emergere in seguito, anche in questi casi di potenziale confusione, l'azione del dicisegno fa in modo che il posto del soggetto e quello del predicato restino perfettamente distinti nelle rispettive funzioni. Anche se la loro composizione può essere oggetto di una continua negoziazione; anche se - come vedremo in 3.2.2. - alcuni tratti semantici del soggetto possono, in certe circostanze, passare a comporre il predicato e viceversa, la possibilità di distinguere le due polarità della relazione predicativa non è mai in discussione.

Un caso analogo a quello delle fotografie è costituito dalle bandierine utilizzate per segnare la direzione del vento. Il verso e la tensione della bandierina sono direttamente causate dalla direzione e dalla forza del vento.

Anche qui, il soggetto della predicazione, il vento, inscrive indicalmente nel segno i caratteri che potranno poi essergli attribuiti. Come nelle fotografie, la parte iconica e indicale della struttura profonda vengono espresse attraverso entità che significano per via indicale; trovano posto sotto forma di indici genuini. 


\section{Ocula $a^{15}$}

Commemorating Charles S. Peirce (1839-1914): interpretive semiotics and mass media

Valerio De Michele, Note per una tipologia dicisegnica

\subsubsection{Dicisegni indicali legisegnici}

Nei dicisegni indicali legisegnici riscontriamo la stessa dinamica, con la differenza che ora le entità indicali chiamate a esprimere la struttura profonda non hanno carattere genuino ma degenerato.

Per illustrare questa tipologia di dicisegni Peirce ricorre a

quella sorta di proposizione che ha come suo predicato il nome di un individuo ben noto; come quando alla domanda "qual è il personaggio rappresentato da questa statua?” si risponde "è Farragut”. (CP 2.265)

Nel dicisegno di risposta "Questo è Farragut", tanto il soggetto quanto il predicato sono coperti da unità indicali: da un dimostrativo e da un nome proprio; due tipici esempi di Subindici.

Concludendo, nei dicisegni indicali, in quelli sinsegnici come in quelli legisegnici, la parte indicale e la parte iconica, che costituiscono la struttura profonda del dicisegno, trovano entrambe espressione attraverso il ricorso a unità segniche di natura indicale (fig. 2).

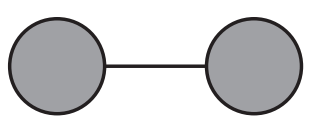

Indice

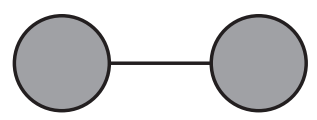

Indice

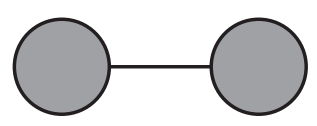

Indice

LIVELLO SUPERFICIALE

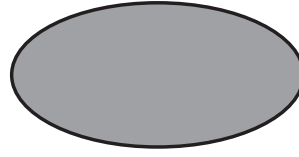

Icona
Figura 2. Struttura del Dicisegno Indicale.

\subsection{I dicisegni simbolici}

Nei dicisegni simbolici la parte indicale e quella iconica della struttura profonda vengono espresse attraverso unità simboliche.

Un esempio potrebbe essere la proposizione: "Il saggio ama la virtù". Qui, tanto il soggetto che il predicato sono resi con entità replicabili; legisegni che non puntano a individui, ma rinviano convenzionalmente a idee generali. Come chiarisce Peirce, il simbolo segna la giunzione, convenzionale, di due idee generali. Tanto dal lato del representamen (funtivo rinviante) che da quello dell'oggetto (funtivo rinviato) troviamo quindi termini generali, type, unità perfettamente delineate nei propri caratteri costitutivi:

Un simbolo è un segno che si riferisce all' oggetto che esso denota in virtù di una legge, di solito un'associazione di idee generali, che opera in modo che il simbolo sia 


\section{Ocula $a^{15}$}

Commemorating Charles S. Peirce (1839-1914): interpretive semiotics and mass media

Valerio De Michele, Note per una tipologia dicisegnica

interpretato come riferentesi a quell'Oggetto. È insomma esso stesso un tipo generale di legge, cioè di legisegno. Come tale agisce attraverso una replica. Non soltanto il simbolo stesso è generale, ma anche l' oggetto al quale si riferisce è di natura generale. (CP 2.249)

La ragione che obbliga entrambi i funtivi del rinvio simbolico ad avere natura legisegnica non è difficile da comprendere: per unire convenzionalmente due entità è necessario definire con precisione cosa viene unito a cosa. È necessario, insomma, operare con type, con idee generali (fig. 3).

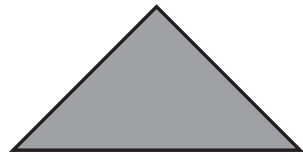

Simbolo

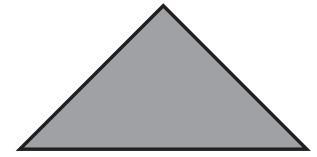

Simbolo

LIVELLO SUPERFICIALE

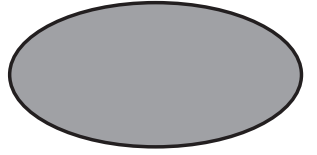

Icona

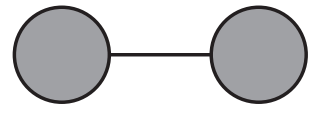

Indice

Figura 3. Struttura del Dicisegno Simbolico.

\section{Rilancio del modello peirciano}

La stringata classificazione dei dicisegni proposta nella Speculative Grammar getta solide basi per un'indagine più approfondita, per l'articolazione di un modello più ampio che possa rappresentarne una coerente prosecuzione.

Il modello peirciano appare incompleto per due ragioni: i) si concentra sulla necessità di esprimere la struttura profonda del dicisegno attraverso il ricorso a unità indicali e simboliche: tralascia, quindi, il possibile ricorso a unità iconiche; ii) suppone che le unità chiamate a esprimere la parte iconica $\mathrm{e}$ la parte indicale di base siano sempre dello stesso tipo: esclude, quindi, che il soggetto e il predicato vengano espressi da segni di specie diversa.

Di seguito, tenterò di ricondurre le due tipologie dicisegniche analizzate a un modello più generale, nel quale: i) per esprimere la struttura profonda si ricorre anche a unità iconiche; ii) la parte iconica e la parte indicale di base possono essere rese anche attraverso tipologie segniche diverse.

Ne derivano nove differenti tipologie dicisegniche. Tra esse troveremo anche le due indicate nella Speculative Grammar. Come si diceva, l'obiettivo di questo modello è integrare la riflessione peirciana, offrirsi come una sua ideale prosecuzione (tabella 1). 


\section{Ocula $a^{15}$}

Commemorating Charles S. Peirce (1839-1914): interpretive semiotics and mass media

Valerio De Michele, Note per una tipologia dicisegnica

\begin{tabular}{|l|c|c|c|}
\hline \multirow{2}{*}{$\begin{array}{l}\text { Saturazione } \\
\text { della parte iconica }\end{array}$} & \multicolumn{3}{|c|}{ Saturazione della parte indicale } \\
\cline { 2 - 4 } & 1. Icona & 2. Indice & 3. Simbolo \\
\hline 1. Icona & 1.1 & 1.2 & 1.3 \\
\hline 2. Indice & 2.1 & 2.2 & 2.3 \\
\hline 3. Simbolo & 3.1 & 3.2 & 3.3 \\
\hline
\end{tabular}

Tabella 1. Matrice delle tipologie dicisegniche.

La tabella mostra che all'interno di un Dicisegno (ad esempio il dicisegno monadico " $X \grave{e} Y$ ”) : i) la PARTE INDICALE, che individua il soggetto della predicazione (" $X$ "), può essere espressa, saturata, tanto iconicamente (come somiglianza) che indicalmente (come co-implicazione diadica) o simbolicamente (come associazione convenzionale di idee generali); ii) allo stesso modo, la PARTE ICONICA, che individua il predicato ("è $Y$ ”), può essere espressa tanto da unità iconiche che indicali o simboliche. Ne risultano nove possibilità combinatorie, nove tipologie dicisegniche.

\subsubsection{Dicisegno Iconico-Iconico}

Nel quale: i) la PARTE ICONICA (predicato) è espressa da un'entità iconica e ii) la PARTE INDICALE (soggetto) è, ugualmente, espressa da un'entità iconica. Insomma, un'unità iconicamente espressa (predicato) viene attribuita a un'unità iconicamente espressa (soggetto).

È il caso:

i) Di alcune etichette alimentari, che presentano un'illustrazione nella quale l'immagine del prodotto (es. una barretta di cioccolato) risulta affiancata da altri elementi (es. una bottiglia di latte e dei chicchi di caffè). Il risultato è un dicisegno interpretabile come: "La barretta di cioccolato (ICONA) è fatta con latte e caffè (ICONA)". Un esempio molto simile è rappresentato dalla pubblicità della pasta Panzani, oggetto della celebre analisi di Roland Barthes. Anche lì troviamo il prodotto, iconicamente espresso, al quale vengono attribuite alcune proprietà, ugualmente espresse in modo iconico. Tuttavia, in tal caso, la complessità del meccanismo retorico e dei processi connotativi attivati dalla fotografia ci porterebbero a perdere di vista il centro della nostra analisi.

ii) Di alcune vignette satiriche, nelle quali il volto di un personaggio noto viene montato su un corpo o presentato in abiti o atteggiamenti fortemente connotati. Ad esempio, in alcune illustrazioni di Forattini, il volto di Veltroni veniva impietosamente montato sul corpo di un verme o le caricature di Berlusconi, Bossi e Fini abbigliate come i tre della banda bassotti. Il risultato è un dicisegno interpretabile come: "Veltroni (ICONA) è un verme (ICONA)". Anche in tal caso tralascio l'analisi dei complessi meccanismi retorici che consentono l'attivazione del dicisegno.

\subsubsection{Dicisegno Iconico-Indicale}

Nel quale: i) la PARTE ICONICA (predicato) è espressa da un'entità iconica e ii) la PARTE INDICALE (soggetto) è espressa da un'entità indicale. Insomma, 


\section{Ocula $a^{15}$}

Commemorating Charles S. Peirce (1839-1914): interpretive semiotics and mass media

Valerio De Michele, Note per una tipologia dicisegnica

un'unità iconicamente espressa (predicato) viene attribuita a un'unità indicalmente espressa (soggetto).

È il caso:

i) Delle fotografie apposte sulle lapidi o delle gigantografie che coprono le facciate dei palazzi in ristrutturazione, nelle quali l'immagine ha la funzione di descrivere un certo evento al quale punta (in quanto posizionata in un certo luogo). Il risultato è un dicisegno traducibile con: "Ciò che è lì (INDICE) è fatto così e così (ICONA)".

Molto vicini a questi casi sono: iia) le ricostruzioni di incidenti stradali mediante modellini che avvengono nei tribunali. Riscontriamo l'allestimento di un diagramma che ha la funzione di descrivere un certo evento al quale punta (in quanto posizionato all'interno di un dibattimento la cui macro-cornice interpretativa prevede il costante riferimento a un certo insieme di fatti). Il risultato è un dicisegno traducibile con: "Ciò che era allora (INDICE) presentava questa dinamica (ICONA)". iib) I quadri con didascalia. Riscontriamo un'immagine che ha la funzione di descrivere una certa persona alla quale punta (grazie all'ipoindice costituito da un nome personale). Il risultato è un dicisegno traducibile con: "Quella persona (INDICE) era così e così (ICONA)".

\subsubsection{Dicisegno Iconico-Simbolico}

Nel quale: i) la PARTE ICONICA (predicato) è espressa da un'entità iconica e ii) la PARTE INDICALE (soggetto) è espressa da un'entità simbolica. Insomma, un'unità iconicamente espressa (predicato) viene attribuita a un'unità simbolicamente espressa (soggetto).

È il caso:

i) Dell'illustrazione, in un libro di chimica, della struttura molecolare di alcune sostanze di uso comune. Troviamo, ad esempio, il reticolo cristallino del cloruro di sodio affiancato alla didascalia "sale da cucina". In questi casi, il diagramma ha la funzione di attribuire proprietà nuove (molto tecniche) a quelle comunemente significate dal simbolo (ampiamente diffuse e derivanti dall'esperienza che, in ambito domestico, possiamo fare di queste sostanze). Il risultato è un dicisegno traducibile con: "Il sale da cucina (simbolo-NOME COMUNE) è fatto così e così (ICONA)".

Molto vicina a questo caso è l'illustrazione che, in un'enciclopedia, si trova associata a un nome comune. Il testo presenta, ad esempio, la fotografia di un persiano affiancata alla didascalia "gatto". In questi casi, l'immagine ha la funzione di attribuire una forma visiva alle proprietà comunemente significate dal simbolo (affrontate con maggiore o minore approfondimento all'interno del lemma corrispondente). Il risultato è un dicisegno traducibile con: "Il gatto (SIMBOLO-NOME COMUNE) è fatto così e così (ICONA)".

È necessario notare che questo ingaggio semiosico della fotografia prevede la narcotizzazione del suo possibile statuto di impronta. Insomma, il processo genetico dell'immagine è ora destituito di ogni pertinenza.

La foto non viene presa in considerazione in quanto indice-causato naturalmente indirizzato verso l'indice-causante, verso l'individuo particolare che l'ha prodotta; si trova invece a svolgere la funzione di un'icona rematica pura: un segno che ha la funzione di esemplificare una serie di proprietà potenzial- 


\section{Ocula $a^{15}$}

Commemorating Charles S. Peirce (1839-1914): interpretive semiotics and mass media

Valerio De Michele, Note per una tipologia dicisegnica

mente riscontrabili in un'ampia classe di individui. Un segno che, di per sé, risulta privo di ogni applicazione indicale e che, di seguito, solo entrando a far parte di un dicisegno indicale riesce a recuperarne una (nel nostro caso attraverso un'unità di ordine simbolico: la parola "gatto").

\subsubsection{Dicisegno Indicale-Iconico}

Nel quale: i) la PARTE ICONICA (predicato) è espressa da un'entità indicale e ii) la PARTE INDICALE (soggetto) è espressa da un'entità iconica. Insomma, un'unità indicalmente espressa (predicato) viene attribuita a un'unità iconicamente espressa (soggetto).

È il caso dell'indicatore del carburante sul display di un'automobile. Alla benzina, espressa attraverso un'icona altamente stilizzata e convenzionale, viene attribuito, di volta in volta, un certo livello quantitativo, espresso attraverso un indice direttamente determinato dal galleggiante presente nel serbatoio. Il risultato è un dicisegno traducibile con: "La benzina (ICONA) è molta o poca (INDICE)".

\subsubsection{Dicisegno Indicale-Indicale}

Nel quale: i) la PARTE ICONICA (predicato) è espressa da un'entità indicale e ii) la PARTE INDICALE (soggetto) è espressa, ugualmente, da un'entità indicale. Insomma, un'unità indicalmente espressa (predicato) viene attribuita a un'unità indicalmente espressa (soggetto).

È il caso, già visto, delle fotografie e delle bandierine segna-vento, ma anche:

i) Delle impronte lasciate nell'argilla da personaggi noti. In questi casi, il nome proprio inciso sotto l'impronta è un indice degenerato, un ipoindice che punta convenzionalmente verso un certo individuo, e il calco della mano è un indice genuino, causalmente determinato dalla presenza fisica della persona in quel dato luogo. Il risultato è un dicisegno traducibile con: "Lucio Dalla (INDICE-DEGENERATO) è stato qui (INDICE-GENUINo)".

ii) Delle orme. Le orme costituiscono un complesso di proprietà indicalmente espresse, all'interno del quale l'interprete è chiamato a distinguere, seguendo le particolari esigenze del caso, quelle che si offrono come soggetto da quelle che si offrono come predicato. Insomma, una stessa costellazione di segni indicali può accogliere, a seconda delle circostanze, differenti polarizzazioni tra PARTE ICONICA e PARTE INDICALE: le stesse proprietà possono ritrovarsi, col variare dei contesti, a fungere tanto da soggetto che da predicato. Vediamo qualche esempio di questa variabilità compositiva della funzione predicazionale.

iia) Torniamo a casa e il nostro fidato cane sembra essere sparito, dopo un po' rinveniamo delle impronte in giardino. Dinanzi alle orme, il nostro obiettivo è verificare se esse siano compatibili con un passaggio recente dell'animale, in modo da scoprire dove fosse diretto. Così, tra le proprietà indicali che compongono le impronte, la forma (indice genuino causalmente connesso all'impressore), la profondità (indice genuino causalmente connesso al peso) e il grado di freschezza (indice genuino connesso al tempo di impressione), risultando tutte compatibili con proprietà note dell'animale (razza, stazza e recente presenza fuori dalle mura domestiche), vengono utilizzate come soggetto, mentre il verso delle impronte (indice genuino connesso alla direzionalità) viene utilizzato 


\section{Ocula $a^{15}$}

Commemorating Charles S. Peirce (1839-1914): interpretive semiotics and mass media

Valerio De Michele, Note per una tipologia dicisegnica

come predicato. Il risultato è un dicisegno traducibile con: "Un cane di razza labrador (INDICE), sovrappeso (INDICE), di recente passato in giardino (INDICE) - quindi probabilmente Fido - andava in quella direzione (INDICE)".

iib) Passeggiamo in un bosco e improvvisamente un formante bianco guizza da un lato all'altro del nostro campo visivo; avvicinandoci al posto in cui lo abbiamo visto passare rinveniamo delle impronte. Dinanzi alle orme, il nostro obiettivo è verificare se esse siano compatibili con il passaggio recentissimo del non meglio precisato animale, in modo da scoprire, finalmente, di cosa si trattasse. Così, tra le proprietà indicali che compongono le impronte, il grado di freschezza (indice genuino connesso al tempo di impressione) e il verso (indice genuino connesso alla direzionalità), risultando tutte compatibili con le proprietà note dell'oggetto (recentissima apparizione e direzione di marcia), vengono utilizzate come soggetto, mentre la forma (indice genuino causalmente connesso all'impressore) e la profondità (indice genuino causalmente connesso al peso), vengono utilizzati come predicati. Il risultato è un dicisegno interpretabile come: "La cosa appena passata di qui (INDICE) e che si muoveva in quella direzione (INDICE) era un cinghiale (INDICE), molto leggero (INDICE), quindi un cucciolo... bianco!”.

\subsubsection{Dicisegno Indicale-Simbolico}

Nel quale: i) la PARTE ICONICA (predicato) è espressa da un'entità indicale e ii) la PARTE INDICALE (soggetto) è espressa da un'entità simbolica. Insomma, un'unità indicalmente espressa (predicato) viene attribuita a un'unità simbolicamente espressa (soggetto).

È il caso dei grandi cartelli informativi posti all'ingresso degli uffici, dei villaggi turistici, dei centri commerciali o dei complessi ospedalieri e industriali. Ad ogni reparto, dipartimento o padiglione, espresso attraverso un simbolo (es. Radiologia, Direzione, Ristoranti), viene attribuita una certa direzione, espressa attraverso un indice toposensitivo: una freccia il cui verso risulta direttamente determinato dal proprio oggetto. Il risultato è un dicisegno traducibile con: "I Ristoranti (sIMBOLO) sono lì (INDICE)".

\subsubsection{Dicisegno Simbolico-Iconico}

Nel quale: i) la PARTE ICONICA (predicato) è espressa da un'entità simbolica e ii) la PARTE INDICALE (soggetto) è espressa da un'entità iconica. Insomma, un'unità simbolicamente espressa (predicato) viene attribuita a un'unità iconicamente espressa (soggetto).

È il caso:

i) Dei depliant illustrativi o dei libretti di istruzioni, nei quali si mette a confronto il modo corretto ed errato di eseguire una certa operazione. Alle Icone che illustrano le due modalità (fotografie o disegni stilizzati) vengono affiancati dei Simboli (scritte, come "Si/No"; "Corretto/Errato"; oppure segni altamente convenzionali, come "Cerchio Verde/ Rosso"; " $X / V$ ").

Il risultato è un dicisegno traducibile con: "L'operazione così condotta (ICONA) è errata (SIMBOLO)".

ii) Dei manifesti affissi per ritrovare qualcuno scomparso. Alla fotografia della persona (ICONA) viene affiancata la scritta: "Scomparso" (simbOLo). 


\section{Ocula $a^{15}$}

Commemorating Charles S. Peirce (1839-1914): interpretive semiotics and mass media

Valerio De Michele, Note per una tipologia dicisegnica

Il risultato è un dicisegno traducibile con: "La persona fatta così e così (ICONA) è sparita (SIMBOLO)”.

\subsubsection{Dicisegno Simbolico-Indicale}

Nel quale: i) la PARTE ICONICA (predicato) è espressa da un'entità simbolica e ii) la PARTE INDICALE (soggetto) è espressa da un'entità indicale. Insomma, un'unità simbolicamente espressa (predicato) viene attribuita a un'unità indicalmente espressa (soggetto).

È il caso:

i) Delle etichette apposte sui barattoli o sui tasti dei dispositivi elettronici per segnalarne il contenuto o la funzione. Abbiamo così un Simbolo (la scritta "Sale/Pepe"; "Play/Rewind") che ha il compito di descrivere un'entità, alla quale punta attraverso il proprio stesso posizionamento. Dicendo meglio, il Simbolo è chiamato a descrivere un'unità che è espressa da un Indice; tale indice, però, presenta uno statuto molto particolare: non si concretizza in un elemento tangibile, ma è una funzione semiosica interamente espressa dal semplice posizionamento dell'entità simbolica (della scritta). Insomma, in questi casi la posizione del simbolo vale come indice toposensitivo; come accade anche nelle già analizzate fotografie funerarie (cfr. 1.2. Dicisegno Iconico-Indicale, punto ii).

Il risultato è un dicisegno interpretabile come: "Ciò che è lì (INDICE) è sale (simbolo)".

ii) Delle proposizioni il cui soggetto è costituito da un nome proprio, un dimostrativo, un pronome o un elemento deittico. Abbiamo così un predicato, formato da un'unità simbolica, che si applica a un soggetto, formato da un'unità sub-indicale. Come, ad esempio: "Quello (INDICE DEGENERATo) è un gatto (SIMBOLO)".

\subsubsection{Dicisegno Simbolico-Simbolico}

Nel quale: i) la PARTE ICONICA (predicato) è espressa da un'entità simbolica e ii) La PARTE INDICALE (soggetto) è espressa, ugualmente, da un'entità simbolica. Insomma, un'unità simbolicamente espressa (predicato) viene attribuita a un'unità simbolicamente espressa (soggetto).

È il caso dei dicisegni simbolici indicati da Peirce: proposizioni nelle quali le entità semiosiche chiamate a comporre tanto il soggetto che il predicato valgono come connessioni di idee generali; rinviano cioè a strutture semantiche intese come complete. Un esempio può essere il già riportato: "Il saggio ama la virtù".

\section{Conclusione}

Le nove tipologie dicisegniche e gli esempi utilizzati per illustrarle rappresentano, come si diceva, un tentativo di avanzare, almeno di qualche passo, nella conoscenza della Grammatica pura, seguendo, per quanto possibile, le orme lasciate da Peirce. È importante sottolineare che gli esempi hanno valore puramente indicativo e che uno stesso oggetto può, in circostanze diverse, contrarre diverse funzioni semiosiche, trovandosi così a "fare il surf" tra più tipologie dicisegniche. 


\section{Ocula $a^{15}$}

Commemorating Charles S. Peirce (1839-1914): interpretive semiotics and mass media

Valerio De Michele, Note per una tipologia dicisegnica

\section{Bibliografia}

Basso Fossali, Pierluigi; Dondero, Maria Giulia

2006 Semiotica della fotografia, Rimini, Guaraldi.

Hénault et Beyaert-Geslin

2004 Ateliers de sémiotique visuelle, Paris, Puf.

Fontanille, Jacques; Dondero, Maria Giulia

2012 Des images à problems, Limoges, Pulim.

Peirce, Charles Sanders

2003 Opere, Milano, Bompiani.

Valerio De Michele è laureato in Scienze della comunicazione all'Istituto universitario Suor Orsola Benincasa di Napoli con una tesi in semiotica dal titolo: La questione dell'iconismo (relatore prof. Stefano Gensini); attualmente sta completando la laurea magistrale in Semiotica presso l’Università di Bologna. 